\title{
Conditional based Edge Detection Algorithm Enhancing the Quality of Clustered Grain Seeds
}

\author{
Honeyily \\ Department of Electronics \& \\ Communication \\ Sri Sai University, Palampur \\ India
}

\author{
Disha Sugha \\ Department of Electronics \& \\ Communication \\ Sri Sai University, Palampur \\ India
}

\author{
Vinay Thakur \\ Department of Electronics \& \\ Communication \\ Sri Sai University, Palampur \\ India
}

\begin{abstract}
In this study, we proposed a method of improving the edge detection of clustered grains (soybean seeds) to identify the actual shape of grain seeds and enhance the edge of seeds with the help of a conditional Sobel operator based edge. We designed methods that improve the edges of clustered soybean seeds from a digital image captured under non-ideal conditions. This is not mandatory, but results will be more reliable if that condition is observed. Basically, we compared some parameter that is energy, mean, median and range of image with respective base paper.
\end{abstract}

\section{General Terms}

Sobel Operator, MATLAB

\section{Keywords}

Edge detection, Clustered seeds, soybean seeds, Digital Image, Image enhancement.

\section{INTRODUCTION}

The clustered grains counting are a very challenging task in visual surveillance, if clustered seeds are counted either manually or by using expensive commercial photo electronic counters, these types of processing are time-consuming, expensive and chances of less accuracy. The techniques proposed here overcome this clustered seed problem by using some technique. We work on the edge of soybean seeds with the help of the Sobel operator. Edge detection is the one of an image processing method for analysis the boundaries of objects within images also extract important feature of an image i.e. corners, lines and curves. The paper aims to develop an effective method for estimating the number of people and locate each individual in a low-resolution image with complicated scenes [3]. Firstly, post-processing steps are performed on background subtraction results to estimate the number of people in a complicated scene, which includes people who are moving only slightly. Second, an Expectation-Maximization (EM) based method has been developed to locate individuals in a low-resolution scene. They presents, a new cluster model used to represent each person in the scene. This method does not require a very accurate foreground contour. Third, the number of people does not require is used as a priori for locating individuals based on feature points. Hence, the methods for estimating the number of people and for locating, individuals are connected. They used the neural network to an estimated number of people, a method based on the EM algorithm to locate individuals. They proposed a system that is work even under far from ideal conditions, including variation in illumination and low contrast between seeds and background. This proposal is basically based on morphological operations largely used in digital image processing they proposed a system under far-from-ideal conditions, including variation in illumination and low contrast between seeds and background [2]. The proposal is mainly based on morphologic operations largely used in digital image processing, in order to make its implementation simple and to keep the computational burden low.

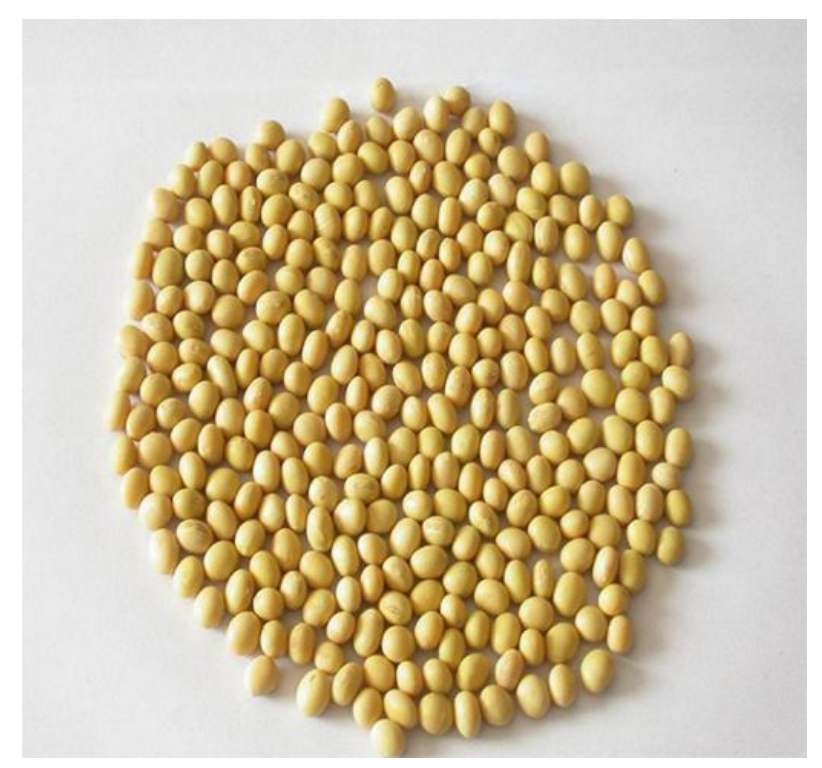

Figure 1: Soybeans seeds

\section{METHODOLOGY}

Edge detection is basically used for image segmentation and data extraction in areas such as image processing, computer vision, and machine vision. The most common edge detection algorithms include Sobel, Canny, Prewitt, Robert, and Fuzzy logic methods; here we used a conditional based Sobel algorithm. The proposed algorithm is evaluated via computer simulation using MATLAB (Matrix Laboratory) simulator. The first step is to capture the image from a distance between 0.5 and 1 meter from the seeds. It is not compulsory but if we capture the image from this way the result will be wellfounded also, the capture must be as vertical as possible to avoid the complication of perspective [2]. The image used here collected from the internet as a reference for the remainder of the paper, because it presents a challenging situation and variation in the illumination.

\subsection{Sobel Operator}

In this study, we worked with the Sobel operator that is used to detect the vertical direction and horizontal direction of an image. It is almost similar to the Prewitt operator of an image. In the case of Sobel edge detection, there are two marks, one mark identifies a horizontal edge and the other mask identifies the vertical edges. The mask which finds the horizontal edges 
that is equivalent to having the gradient in equivalent to taking in the gradient in a horizontal direction.

\subsection{Steps in program development}

- Capture the image (soybean seeds).

- The Algorithm is converting RGB to a gray image.

- CLAHE (Contrast Limited Adaptive Histogram Equalization), basically used on small part (tiles) of an image and used to avoid over-saturation in the homogeneous area like the background.

- Adaptive thresholding applied to the image: it computes a locally adaptive threshold for the 2-D grayscale image or 3-D grayscale. It chooses the threshold based on local mean intensity in the neighborhood of each pixel. Binary conversion applied to the image.

- Reversing the binary image.

- Appling filter to enhance the shape.

- Appling filter to remove noise

- Dilation applied: It helps to join all areas with high derivative value and after that erodes the image back to the original image. The white region plays a role like a mask inside the picture.

- Image enhancement

- The Boundary of clustered seeds

- The Threshold applied to the image

- Removing Noise

\section{SIMULATED RESULTS}

Therefore the output of the proposed algorithm shown in the below:

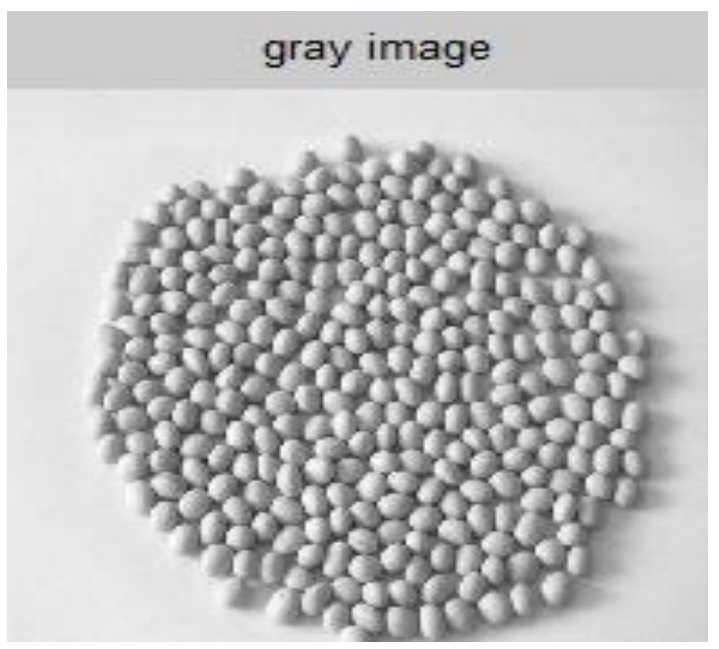

Figure: 2

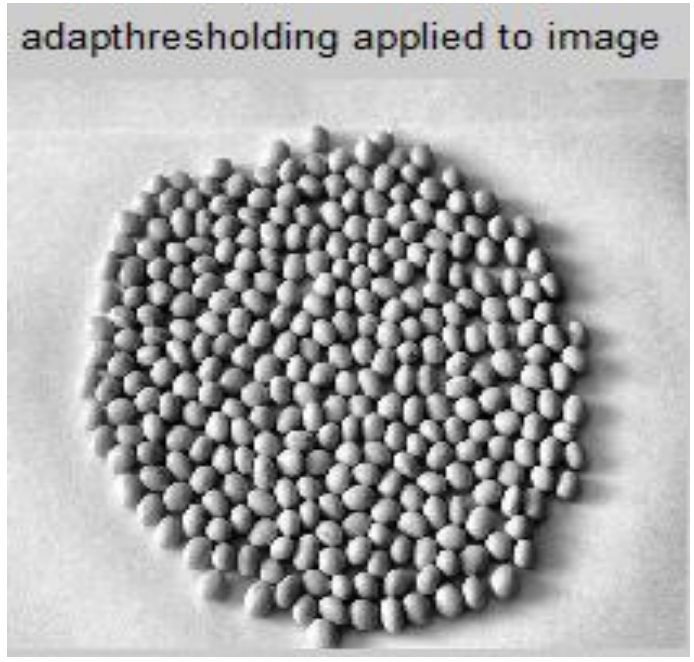

Figure: 3

Binary conversion output image

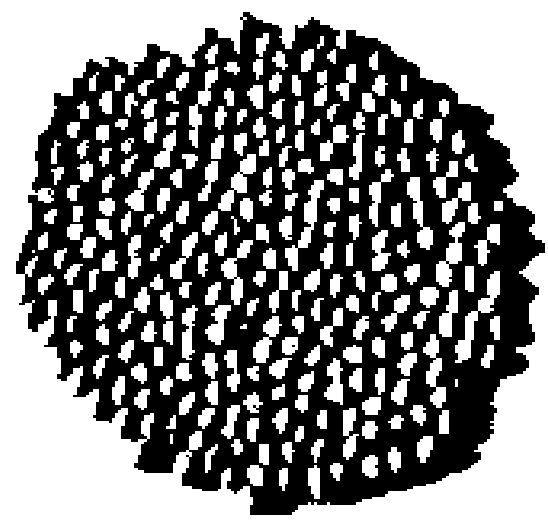

Figure: 4

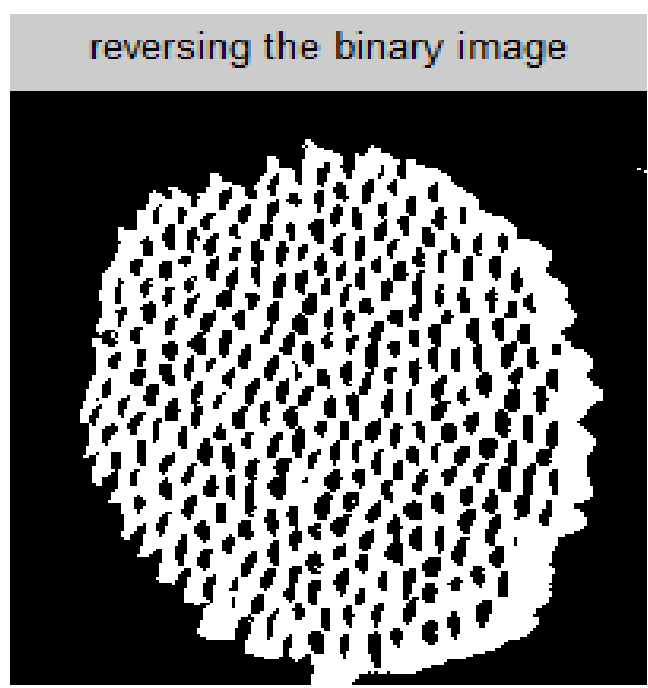

Figure: 5 


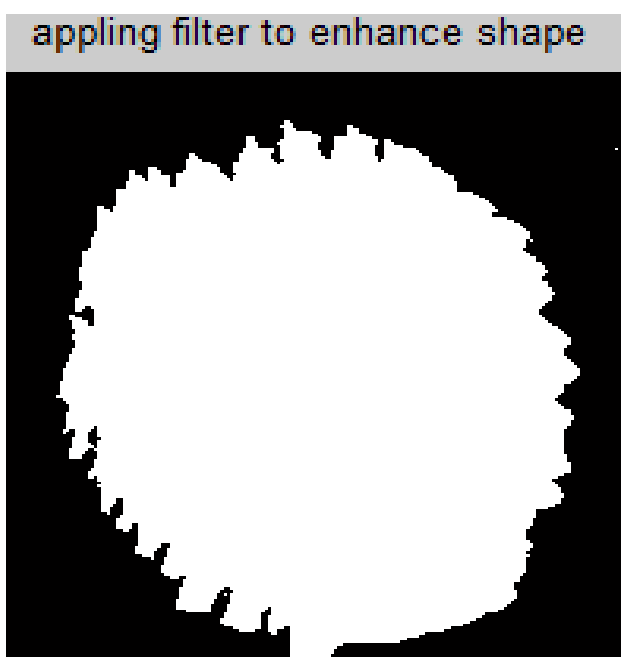

Figure: 6

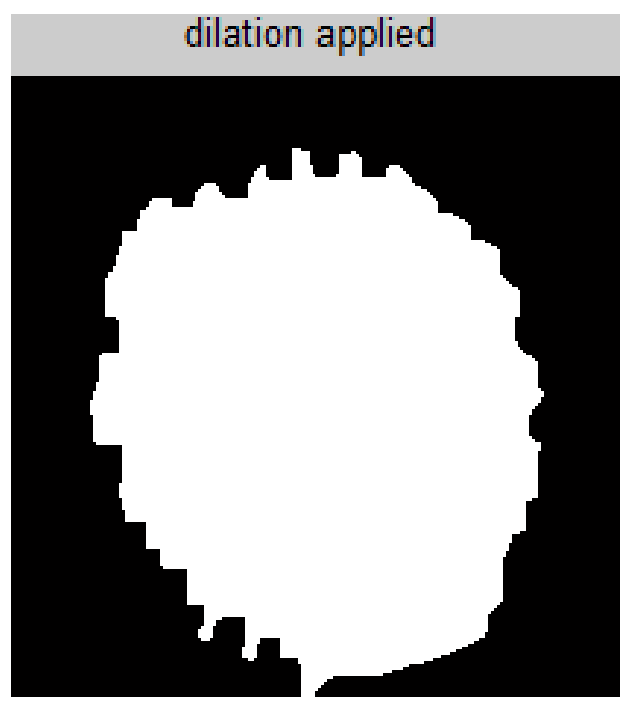

Figure: 7

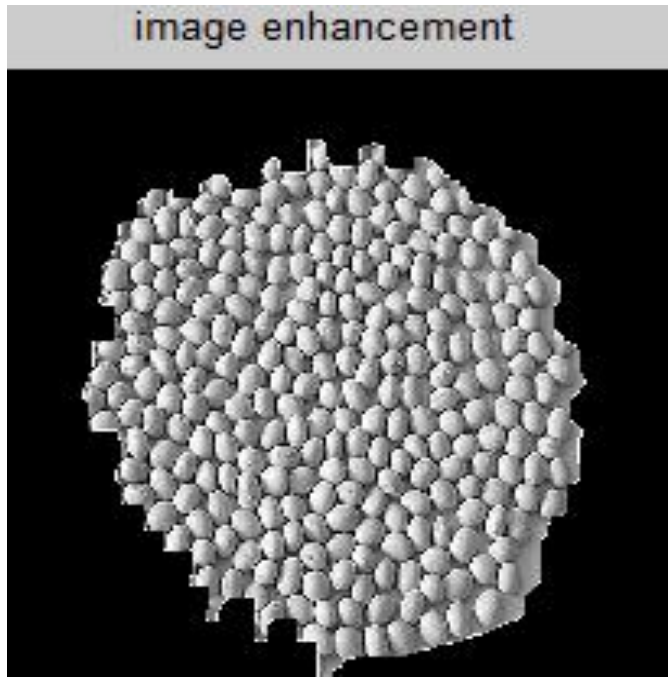

Figure: 8

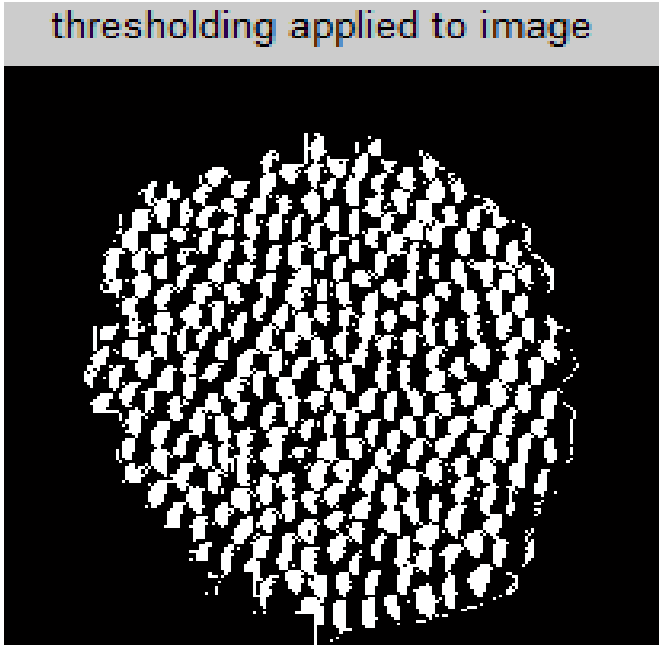

Figure: 9

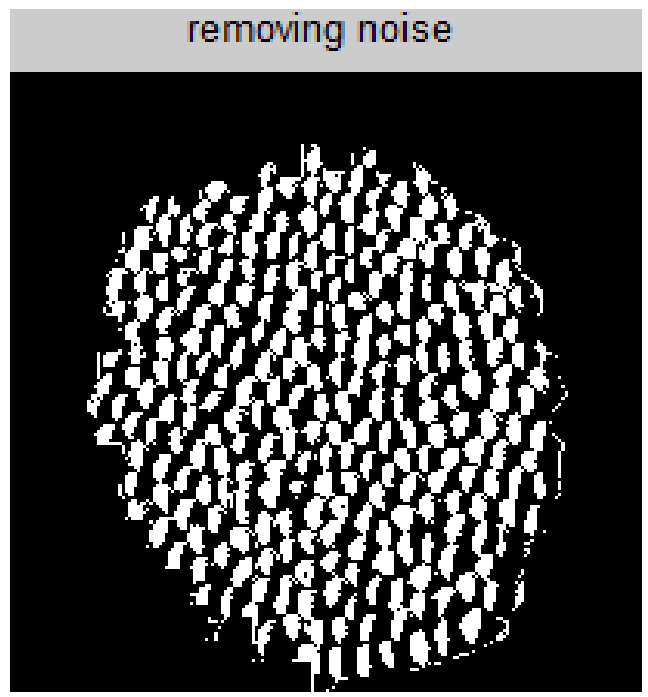

Figure: 10

\section{CONCLUSION}

On the basis of previous analyses, we compared some parameters (i.e. energy, mean, median and range) with the base paper. Our aim is here, to enhance the quality of clustered seeds by using a conditional Sobel algorithm and to obtain the output with their actual shape. According to previous approaches they represented the edge of the seeds in the shape of a circle; in this study, we overcome this by using some advanced approaches. 


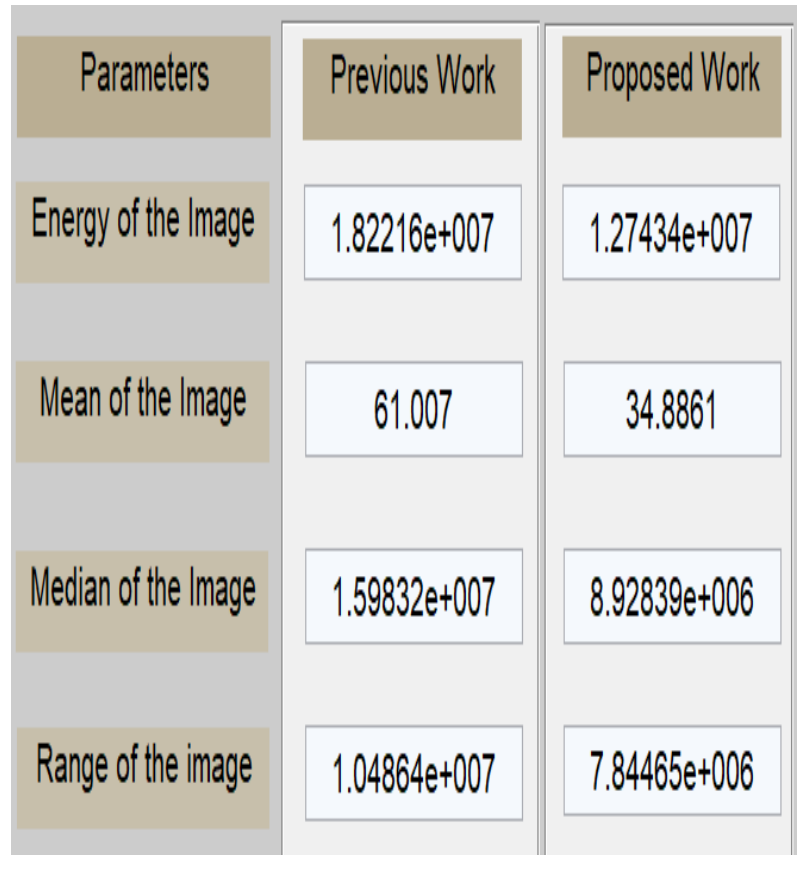

Figure: 11

\section{REFERENCES}

[1] Varun Sanduja and Rajeev Patial 2012 Sobel Edge Detection using Parallel Architecture based on FPGA. International Journal of Applied Information System.

[2] Jayme Garcia Arnal Barbedo 2012 Counting Clustered Soybean Seeds. $12^{\text {th }}$ International Conference on Computational Science and Its Application.

[3] Ya-Li Hou and K. H. Pang 2011 People Counting and Human Detection in a Challenging Situation. IEEE Transactions on System, Man and Cybernetics- Part A: Systems and Humans.

[4] P. Zhao and Y. Li 2009 Grain Counting Method Based on Image Processing. Internationals Engineering and Computer Science.

[5] C. M. Costa and S. Yang 2009 Counting pollen grains using readily available, free image processing and analysis software. Annals of Botany.

[6] J.M. Bewes, N. Suchowerska and D.R. MeKenzie 2008

Automated cell colony counting and analysis using the circular hough image transform algorithm (ChiTA). Physics, Medicine and Biology. 